\title{
Reconstruction of cosmic and beam-halo muons with the CMS detector
}

\author{
Chang Liu ${ }^{1, a}$, Norbert Neumeister ${ }^{1}$, Nicola Amapane ${ }^{2}$, Cristina Fernandez Bedoya ${ }^{3}$, Riccardo Bellan ${ }^{2,4}$, \\ Philipp Biallass $^{5}$, Sara Bolognesi ${ }^{2}$, Gianluca Cerminara ${ }^{2}$, Mary-Cruz Fouz Iglesias ${ }^{3}$, Marina Giunta ${ }^{6}$, \\ Luigi Guiducci $^{6}$, Kerstin Hoepfner ${ }^{5}$, Stefano Lacaprara ${ }^{7}$, Gianni Masetti ${ }^{6}$, Anna Meneguzzo ${ }^{8}$, Pierluigi Paolucci ${ }^{9}$, \\ Jesus Puerta Pelayo ${ }^{3}$, Riccardo Travaglini ${ }^{6}$, Marco Zanetti ${ }^{4}$, Carlos Villanueva ${ }^{3}$ \\ ${ }^{1}$ Department of Physics, Purdue University, West Lafayette, IN, USA \\ ${ }^{2}$ Università di Torino e Sezione dell'INFN, Torino, Italy \\ ${ }^{3}$ CIEMAT, Madrid, Spain \\ ${ }^{4}$ CERN, Geneva, Switzerland \\ ${ }^{5}$ Physics Institute III A, RWTH Aachen University, Aachen, Germany \\ ${ }^{6}$ INFN and University of Bologna, Bologna, Italy \\ ${ }^{7}$ INFN, Legnaro, Italy \\ ${ }^{8}$ INFN and University of Padova, Padova, Italy \\ ${ }^{9}$ INFN, Naples, Italy
}

Received: 6 February 2008 / Revised: 3 July 2008 / Published online: 30 July 2008

(c) Springer-Verlag / Società Italiana di Fisica 2008

\begin{abstract}
The powerful muon and tracker systems of the CMS detector together with dedicated reconstruction software allow precise and efficient measurement of muon tracks originating from proton-proton collisions. The standard muon reconstruction algorithms, however, are inadequate to deal with muons that do not originate from collisions. This note discusses the design, implementation, and performance results of a dedicated cosmic muon track reconstruction algorithm, which features pattern recognition optimized for muons that are not coming from the interaction point, i.e., cosmic muons and beam-halo muons. To evaluate the performance of the new algorithm, data taken during Cosmic Challenge phases I and II were studied and compared with simulated cosmic data. In addition, a variety of more general topologies of cosmic muons and beamhalo muons were studied using simulated data to demonstrate some key features of the new algorithm.
\end{abstract}

\section{Introduction and motivation}

The efficient and accurate detection of muons and the reconstruction of their momenta with high precision over a large range of muon energies are crucial for the LHC physics program. The Compact Muon Solenoid (CMS) experiment [1]

a e-mail: chang.liu@cern.ch at the LHC provides excellent muon identification and reconstruction capabilities. A large superconducting solenoid with a $4 \mathrm{~T}$ magnetic field provides strong bending power, allowing a precise measurement of the momentum. A complex muon system has been designed that consists of 3 different types of detectors, sandwiched between layers of the iron return yoke. Centrally-produced muons are detected in the silicon tracker, the calorimeters, and the muon system.

In addition to centrally-produced muons, particles that do not originate from $p-p$ collisions, such as cosmic muons and beam-halo muons, can be recorded by the CMS detector. However, the detection and reconstruction of cosmic and beam-halo muons are different from that of muons from $p-p$ collisions. Cosmic muons are the most abundant particles originating from cosmic rays at sea level [2]. Beamhalo muons are machine-induced particles that travel along the beam line. Although in physics analyses these types of muons are generally considered as sources of background, they can be used for detector alignment, calibration, and detector performance validation. The efficient reconstruction of cosmic and beam-halo muons is especially important for the commissioning phase of the detector.

Since the standard muon reconstruction software has been optimized to identify and reconstruct muons originating from $p-p$ collisions, a different optimization must be carried out to reconstruct effectively the muons coming from outside the detector. A dedicated cosmic muon reconstruction software was developed and the performance was tested 
with real data taken during the CMS Magnet Test and Cosmic Challenge (MTCC) [3]. Unlike muons from collisions, which are moving radially outward, cosmic muons arrive at the detector from random directions and at random times. They can traverse either both hemispheres or only a small part of the detector depending on their energy and direction. Figure 1 illustrates the different topologies of muons coming from outside and from $p-p$ collisions. In some cases, as indicated in Figs. 1(b) and (c), the standard muon reconstruction algorithm can reconstruct a cosmic muon, but the muon will be recognized as 2 separate tracks. Cosmic muons arriving at the detector in coincidence with $p-p$ collisions are a potential background for the physics processes. Distinguishing them from real muon events is crucial for many physics analyses. In addition, reconstructing such muon trajectories provides an important tool for aligning detector components and studying trigger and reconstruction efficiencies, especially during the initial data taking period [4].

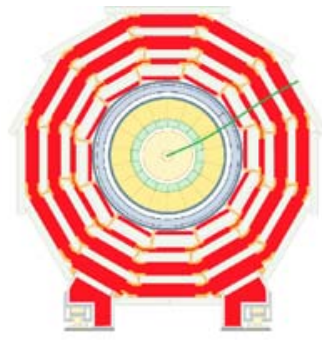

(a)

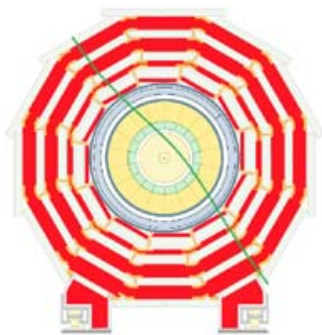

(c)

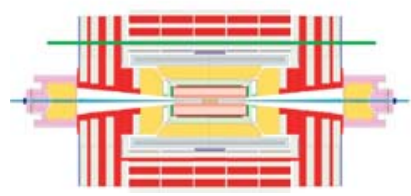

(e)

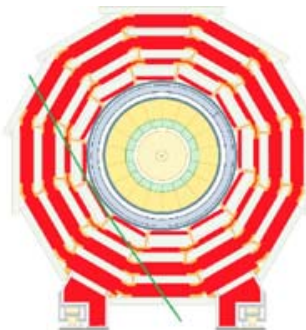

(b)

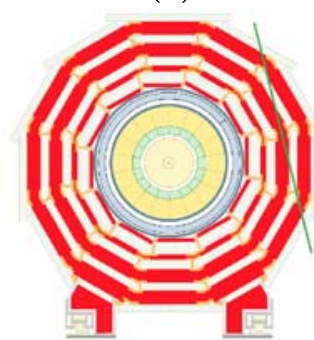

(d)

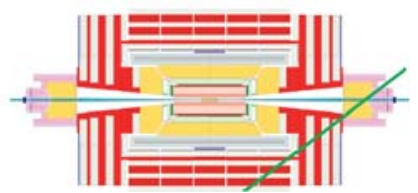

(f)
Fig. 1 Illustration of the differences among muons from $p-p$ collisions, different types of cosmic muons, and beam-halo muons. (a) Muons from collisions always propagate from the center to the outside and the pattern is well-defined; (b) Cosmic muons can penetrate the detector and leave signals in opposite hemispheres of the muon system; (c) Cosmic muons can leave signals in the tracker system and opposite hemispheres of the muon system; (d) Cosmic muons can enter the detector and leave without passing through all muon detector layers; (e) beam-halo muons can penetrate the detector and leave signals in both endcap regions; (f) Cosmic muons can enter the endcap region and leave from the barrel region of the detector (or vice versa, in the upper part of the detector)
Muon reconstruction as implemented in the official CMS software framework is performed in 3 stages: local pattern recognition within each muon chamber, standalone reconstruction that builds tracks within the muon system, and global reconstruction that builds tracks using data from the muon system and the silicon tracker. Already at the level of local reconstruction in the muon system, cosmic muons and beam-halo muons should be treated differently. For example, in the barrel drift tube muon system, drift times are recorded and transformed to local positions for further reconstruction. The latencies of different drift tube chambers and readout electronics are different and depend on the location of the muon track and on its time of arrival within the (arbitrary, in the case of cosmic muon) bunch crossing (BX) window defined by the trigger. Since cosmic muons arrive randomly in time, a specific calibration process is carried out as discussed in [5, 6]. However, the local reconstruction of cosmic muons is very detector specific and is out of the scope of this study. In this article, we focus on the standalone and global muon reconstruction steps by presenting the limitation of the standard reconstruction algorithms and proposing an alternative reconstruction algorithm for cosmic and beam-halo muons. The standard algorithms are designed with the assumption that muons are coming from the interaction point and the direction of the energy flow of trajectories is always out-going from the center of the detector. Pattern recognition based on this assumption is not suitable for the reconstruction of muons coming from outside the detector, except for some special cases when the direction of cosmic muons is pointing to the interaction point. To correctly and efficiently reconstruct cosmic muons and beam-halo muons, the cosmic muon reconstruction algorithm assumes that muons are coming from outside, and is optimized by utilizing properties of cosmic muons and beam-halo muons as discussed below.

The new cosmic muon reconstruction software has been released and is available to the CMS community as a part of the official CMS software releases. During the MTCC the new cosmic muon reconstruction software was employed successfully to reconstruct cosmic muons traversing a full slice of the CMS detector. Although the initial motivation of the design was to reconstruct cosmic muons, the reconstruction algorithm can also be applied to beam-halo muons.

The performance of the new cosmic muon reconstruction algorithm was studied using simulated data from a dedicated Monte Carlo cosmic muon generator [5] as well as data taken during MTCC. Since the data collected from MTCC reflect only a subset of all use cases indicated in Fig. 1, several scenarios were studied with simulated cosmic and beam-halo event samples to demonstrate some key features of the new reconstruction algorithm. 


\section{The CMS muon system}

\subsection{Detector layout}

The CMS muon system [7] is composed of 3 independent subsystems, as illustrated in Fig. 2. In the barrel region $(|\eta|<0.8)$, drift tube (DT) detectors are installed, while cathode strip chambers (CSC) are used in the endcap regions $(1.2<|\eta|<2.4)$. In the intermediate ("overlap") region $(0.8<|\eta|<1.2)$, chambers of both detectors are crossed by a muon track from the interaction point. Resistive plate chambers (RPC) are installed in the $|\eta|<1.6$ region, covering both the barrel and the endcaps. RPCs have limited spatial resolution, but good time resolution, thus can provide excellent bunch crossing identification. The barrel muon system is arranged in 5 wheels along the $z$-axis, where each wheel is divided into 12 sectors and 4 stations called (from innermost to outermost) MB1, MB2, MB3, and MB4. Each station consists of 12 chambers, except for MB4, which has 14 chambers. The endcap muon system is arranged in 4 stations at each end of the detector. They are numbered from ME1 to ME4 in order of their absolute values of $z$-position. The innermost CSC stations are composed of 3 concentric rings, while the other stations are composed of 2 rings only. Each ring consists of 18 or 36 trapezoidal chambers.

\subsection{Readout for cosmic and beam-halo muons}

The synchronization among sectors for cosmic and beamhalo muons is different from that for muons from $p-p$ collisions because cosmic and beam-halo muons move in different directions and arrive randomly in time. The typical time interval for a cosmic muon traversing the entire CMS detector from top to bottom is around $50 \mathrm{~ns}$ (2 BXs). Beamhalo muons arrive at the interaction point at the same time as the beam (up to a few nanoseconds). However they pass through one end of the detector before the other. The typical time interval for a beam-halo muon to traverse the CMS detector from one endcap to the other is less than $70 \mathrm{~ns}$, thus within 3 BXs. A piece of the track in one hemisphere can be used to trigger the event, while the other piece is usually suppressed by the trigger, but can still be read out in the same event. For the DT system, the measured drift time for each recorded hit in the drift tube cells is buffered within a programmable time window of the order of 20-30 BXs. For the CSC system, the signals of deposited charges is buffered in switched capacitor arrays in 8 time bins, where the width of each time bin is $50 \mathrm{~ns}$. When a trigger fires, the counts of the deposited charges in the 8 time bins are stored as a vector of integers [7]. Therefore, with a well-defined cosmic or beam-halo trigger strategy and local calibration, DT and CSC systems are able to take a "snap shot" of the full trajectory of a cosmic or beam-halo muon that includes 2 track pieces on opposite hemispheres in an event.

A study of the trigger and BX assignment for beam-halo and cosmic muons is presented in [8].

\section{Algorithm}

Muon reconstruction in CMS software is performed in 3 stages: local reconstruction, standalone reconstruction, and global reconstruction. Local reconstruction builds hits and segments within each muon chamber. Standalone reconstruction generates trajectory seeds and builds standalone muon trajectories using only information from the muon detectors. Global muon reconstruction takes the standalone muon trajectories, associates them with corresponding hits or tracks in the silicon tracker, and builds global muon trajectories with information from both muon and tracker systems. The assumption that muons come from the interaction
Fig. 2 Layout of the CMS muon system

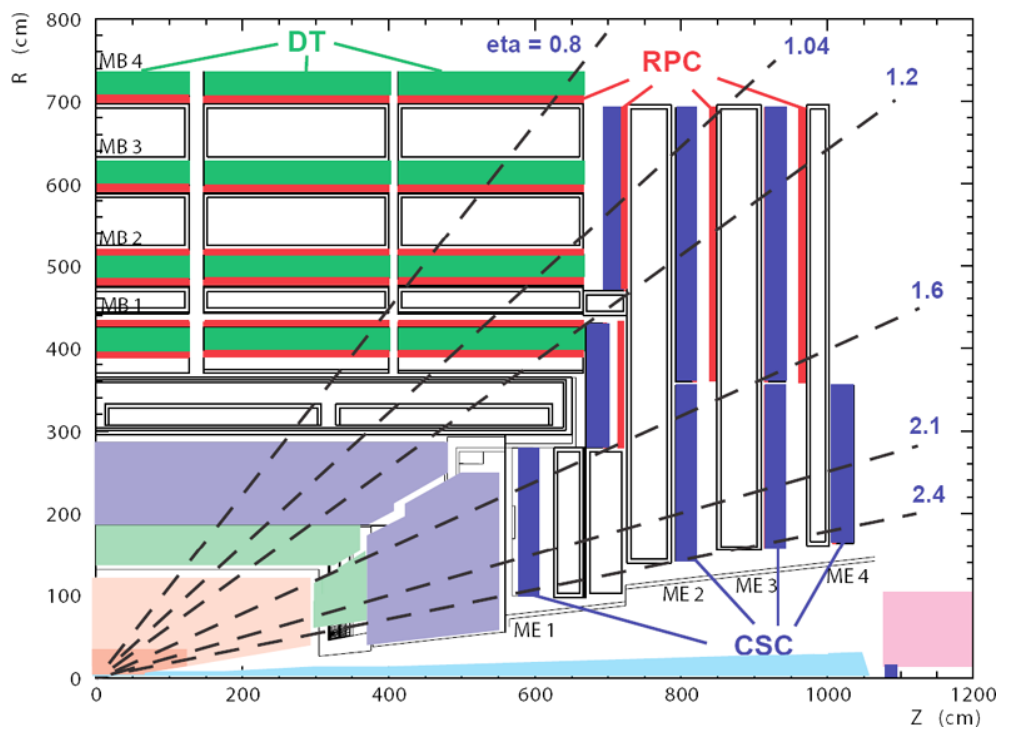


point is implemented in all 3 steps and has to be turned off when dealing with cosmic or beam-halo muons. The new cosmic muon reconstruction software follows the same reconstruction structure and uses as many common tools as possible, specializing and optimizing each individual component to adapt to the properties of cosmic and beam-halo muons.

Here the strategies of standalone and global reconstruction for cosmic muons are discussed. Since the track segments locally reconstructed in each chamber are built without any vertex constraint, the first step of the muon reconstruction consists in building trajectory seeds starting from them.

\subsection{Seeding}

Standalone muon reconstruction starts with locally reconstructed hits and track segments from all muon chambers. The first step of standalone muon reconstruction is to generate trajectory seeds. A trajectory seed contains a state vector (track position, momentum, and errors) associated with 1 or a few DT or CSC hits or segments. Trajectory seeds are the input to the following trajectory building step, and are usually the starting point to build a trajectory. In the standard standalone muon seed generation algorithm, the state vector of a seed is estimated by a parameterization according to the relative positions of selected segments and the interaction point. In the current implementation of cosmic muon seed generation, there are several modifications with respect to the standard algorithm. Each muon trajectory seed is built from exactly 1 DT or CSC segment. Segments located higher (holding a larger $y$ value in the CMS reference coordinates [7]) in the DT system are preferred to lower ones for consideration of the place where cosmic muon trajectories start, while the segments located in the outermost endcap layers are preferred to inner ones for beam-halo muons. The direction of the trajectory state vector is determined by the direction of the segment, which excludes the parameterization involving the interaction point. The momentum direction for all cosmic muons is set to be downward because most cosmic muons travel this way. However, only segments with measurements in all space coordinates can ensure a qualified cosmic trajectory seed. While segments without information in one coordinate are used in standard seed generation, in cosmic seed generation they are used only when there is no better segment available in the event.

\subsection{Navigation}

The next step is to identify the allowed path of a muon trajectory starting from the trajectory seed. The tracking detectors in CMS can be abstracted as a series of layers: cylinders in the barrel region and disks in the endcap region. A muon

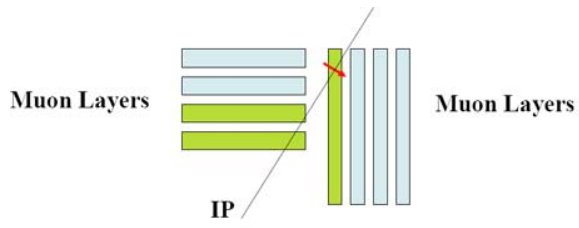

(a)

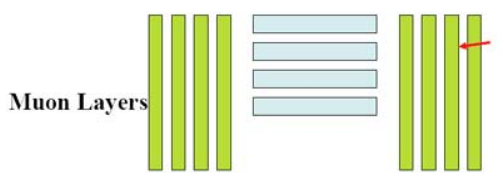

(c)

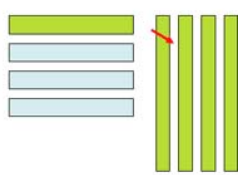

(b)
Fig. 3 Illustration of the differences between the 2 navigation methods using a simplified geometry. (a) Standard Navigation, (b) Direct Navigation, (c) Direction Navigation for beam-halo muons. The muon layers marked as green (or dark shadow) are the chosen compatible layers for a given trajectory state marked as an arrow

trajectory starting from the interaction point crosses the layers in a well-defined sequence, i.e., always in an inside-out sequence and within a small $\eta$ window. For any given trajectory state, the so-called "navigation" algorithm decides on the compatible layers, which are defined as the possible layers that the trajectory may pass through. The navigation used for cosmic muons is fundamentally different from the one used for the reconstruction of muons from collisions. The difference is illustrated by the examples in Fig. 3. Taking a trajectory state as indicated by the arrow with the direction pointing to bottom-right, for example, the standard method checks the $\eta$ of the position of the trajectory state, and chooses all muon layers that cover or intersect the $\eta$ window around the state. The order of compatible layers is determined by their relative distances with respect to the interaction point. The new direct muon navigation algorithm used in cosmic muon reconstruction does a straight-line extrapolation along the direction of the trajectory state from its position, and chooses all muon layers that intersect or are close to it. The order of the compatible layers is determined by their relative positions with respect to the trajectory state's position and direction. In Fig. 3, the layers filled with green (or dark shadow) color are determined as compatible layers with the given trajectory state by 2 different methods. For beam-halo muons, the direct navigation method picks up all layers in both endcaps and skips all barrel layers, as indicated in Fig. 3(c). The direct muon navigation method can also be used for muons from $p-p$ collisions with similar performance to the standard one.

\subsection{Trajectory building in the muon system}

Cosmic muon reconstruction builds trajectories starting from trajectory seeds, as well as hits and segments built in different muon subsystems. A forward fit starts from the trajectory state extracted from the seed. All compatible layers 
of the trajectory state are looped over to grow a trajectory using the Kalman-filter technique [9]. For each compatible layer, a trajectory state is predicted according to the trajectory state on the previous layer or in the seed, then the predicted trajectory state is compared with existing measurements on the current layer. When a measurement is found compatible with the state, a new updated trajectory state with combined information is created and added to the trajectory. After looping over all compatible layers, a backward refit is applied using the same method.

When a track segment is built from its contributing hits during muon local reconstruction, the segment is represented by a straight line and the information inside the chamber is lost. To grow a more precise track, the individual reconstructed hits contained in selected track segments instead of the track segments themselves are used during the backward refit. The hits in each track segment are put into the trajectory in an order called the navigation direction. The navigation direction is used locally within each chamber and is defined as outside-in or inside-out according to the distance with respect to the interaction point (radius in the barrel region and absolute $z$ component value in the endcap region). The navigation direction never changes during a fit or refit for a muon from $p-p$ collisions. For cosmic muons, the navigation direction may change when the location changes, e.g., when a muon enters from endcap to barrel, as illustrated in Fig. 1(f). Assuming the cosmic muon is coming from above and the fitting is performed along the momentum, the navigation direction is outside-in in the endcap region, but becomes inside-out in the barrel region. The navigation direction is carefully checked in cosmic muon trajectory building when the location changes. For beam-halo muons, the navigation direction is set as outside-in in one endcap and inside-out in another. A configurable $\chi^{2}$ threshold is used to reject bad hits, mostly due to showering, delta rays, and pair production. In case no matching hits or segments are found (e.g., due to detector inefficiencies, geometrical cracks, or hard showering), the search is continued in the next compatible layer. The state is propagated from one layer to the next, taking into account the muon energy loss in the material, the effect of multiple scattering, and the nonuniform magnetic field in the muon system. After the forward fit and backward refit, the track parameters (5 numbers to determine a helix and their uncertainties) are calculated from the extrapolation of the most accurate trajectory state to the interaction point, although the state closest to the interaction point is not as meaningful for cosmic muons as it is for muons from collisions.

A special algorithm is needed to build those trajectories that are referred to as traversing muons in the following text (see Fig. 1(b)). Traversing cosmic muons come from outside, traverse the detector, and leave from the other side, passing through some cylinders in the barrel region twice.
When grouping all compatible layers together, those cylinders appear in the list twice. Thus the next layer of the innermost cylinder can be the cylinder itself in a middle refit step. In this case, when the trajectory state of the current layer is propagated to the next layer, the predicted state is on the same surface and is the same as the original state, the trajectory building cannot be continued to the other hemisphere. Therefore, the compatible layers in the 2 hemispheres should be determined separately. After the fit in the first hemisphere is finished, if there are enough unused hits remaining in the detector and the trajectory ends up in an inner cylinder, the mechanism of building traversing muons is turned on. The last trajectory state of the trajectory in the first hemisphere is propagated inward to construct a trajectory state on a virtual plane, which is perpendicular to the direction of last trajectory state and passing through the origin. The trajectory state is used as the seed for the rest of the trajectory building process. Compatible layers of this trajectory state are iterated to update the trajectory with measurements. The trajectory building is then continued in the other hemisphere with the opposite navigation direction. To avoid the trajectory state propagating back to the first hemisphere, the propagation direction has to be explicitly specified as "along momentum" or "opposite to momentum" according to the location of the existing half of the trajectory. While propagating between layers within the same hemisphere, the propagation direction can be automatically decided in CMS software.

As the final step of trajectory building, the trajectories are smoothed to get better track parameters. When smoothing a trajectory, the propagation from the last trajectory state in one hemisphere to a hit located in another hemisphere is treated in a special way, because the propagation direction has to be determined as along momentum or opposite to momentum by the relative position of the destination plane and the direction of the starting state, when the destination plane is far from or almost parallel to the direction of the starting trajectory state, it is likely that the propagation direction is estimated incorrectly and thus the propagation to the destination plane fails. In this case, several virtual planes are built between the starting state and the next hit, and the trajectory state is propagated one by one, as illustrated in Fig. 4.

\subsection{Global cosmic muon reconstruction}

Global cosmic muon reconstruction starts from tracks built by the standalone cosmic muon reconstruction and extends the track to include hits from the silicon tracker, if they exist.

The tracks in the tracker system are built by another track reconstruction algorithm dedicated to reconstruct cosmic muons within the tracker system [10]. For each standalone muon track, only the tracks that match its momentum direction within an $\eta-\phi$ region around the cosmic muon 


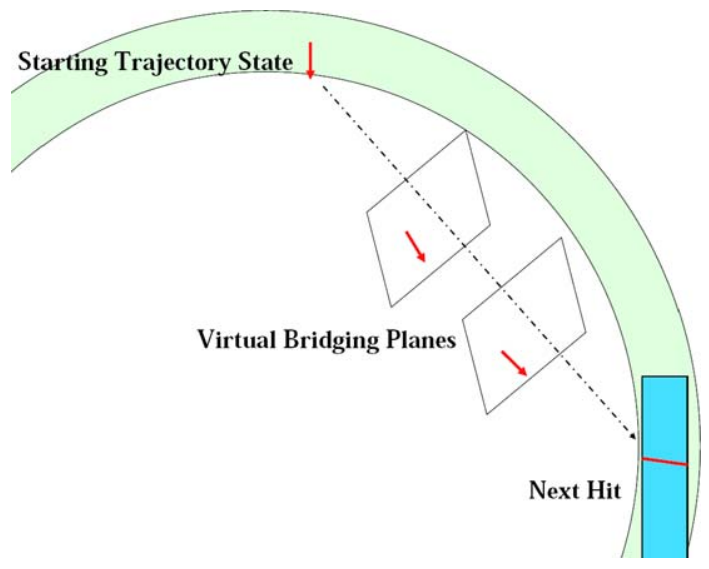

Fig. 4 Illustration of propagation inside the magnetic coil

track are selected. Compared with global muon reconstruction, the matching step is looser.

In the next step, all hits in the standalone muon and its matched tracker track are sorted. Each cosmic muon trajectory is divided into 2 parts in 2 opposite hemispheres of the muon system. The part containing the injection point with higher latitude should be the place where the cosmic muon entered the detector and hits in this hemisphere are sorted as outside-in, while hits in the other part are sorted as insideout. The tracker hits are inserted between the 2 parts of muon hits with an order sorted by their vertical positions. After the order of hits is decided and a starting trajectory state is chosen from the muon track, another Kalman fitting process is applied to iterate over the hits along and opposite to momentum. This step is relatively fast since all hits are wellconfined and no additional pattern recognition is needed. In this case, the number of hits in a single muon track can be more than 100, which allows a very precise measurement.

Given the small size of the tracker system and the random distribution of cosmic muons, only a small fraction of cosmic and beam-halo muons can leave hits in both the tracker and the muon system. After LHC start-up, the hit multiplicity inside the tracker will make it difficult to distinguish cosmic and beam-halo muon hits from those from collisions. Therefore, global cosmic muon reconstruction can only be applied to some specific commissioning phases before LHC collisions.

\subsection{Determination of the trajectory direction}

Generally, there are 2 methods to determine the direction of a trajectory: measuring the time-of-flight and measuring the energy loss of a muon passing through the detector. Measuring the time-of-flight is feasible with the CMS detector by using different muon subsystems, which can provide excellent time resolution of a few nanoseconds [7]. The timing information collected by each chamber can be stored in the muon hits and segments. The direction of a trajectory thus can be determined according to its associated hits.

Another method for estimating the direction of a trajectory is to refit the trajectory in both propagation directions taking into account the energy losses. Since the reconstruction is able to build the trajectory even if the initial propagation direction is wrong, the propagation direction can be determined after the trajectory has been built. One can refit the trajectory in its original propagation direction and get one normalized $\chi^{2}$ value, then flip the momentum of the starting trajectory state, refit the trajectory with the opposite propagation direction, and get a second normalized $\chi^{2}$ value. Although the hits included in the 2 trajectories are the same, the opposite propagation directions take different energy loss increments and render different predicted states on each step. Therefore the overall normalized $\chi^{2}$ values of the final trajectories are different. The trajectory with the smaller $\chi^{2}$ should have the correct propagation direction. However, the method requires more computation time and the effectiveness heavily depends on the track quality. Because the curvature measurement of beam-halo muons is often inaccurate since they travel parallel to the magnetic field, only about $60 \%$ of directions estimated by this method are correct.

In the current implementation, the 2 methods described above are not adopted. The trajectory direction is estimated from the trajectory itself according to properties of cosmic and beam-halo muons. It is usually safe to assume that all cosmic muons are coming from above. Beam-halo muons enter the detector with a direction that is almost parallel to the beam line. The transverse momentum $p_{\mathrm{T}}$ at the starting position is very small and the momentum projection in the $z$ direction $p_{z}$ is large. After passing through several layers of the detector, $p_{z}$ substantially decreases, while $p_{\mathrm{T}}$ decreases much less or can even increase due to multiple scattering and the nonuniform magnetic field in the endcap region. Therefore, one can determine the direction of beam-halo muons by comparing the angle with respect to the $z$-direction of the momenta of trajectory states in the 2 ends. Figure 5 shows the difference between the $\eta$ values of the momenta of first and last trajectory states. About $80 \%$ of reconstructed beamhalo muons have the property $\eta_{\text {first state }}>\eta_{\text {last state }}$, which verifies the effectiveness of the method. In addition, the direction is obvious for those beam-halo muons that do not reach the second endcap.

\section{Data}

To study the efficiency of the algorithm, both real data taken during the Cosmic Challenge and simulated cosmic and beam-halo samples were used. 


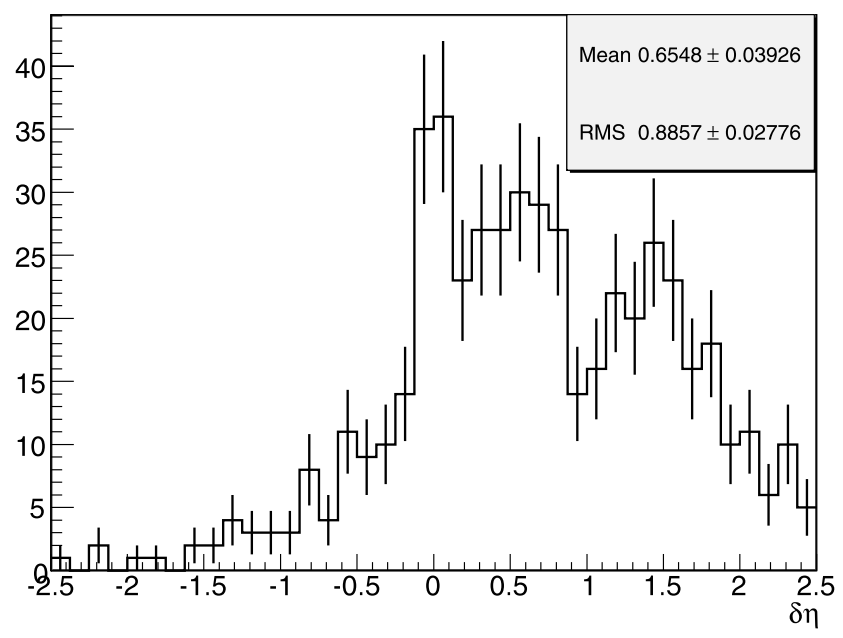

Fig. 5 Distribution of $\eta_{\text {first state }}-\eta_{\text {last state }}$ for reconstructed beam-halo muons

\subsection{MTCC data}

In the second half of 2006, the CMS superconducting magnet was successfully operated at up to $4 \mathrm{~T}$ for the first time. The magnet test campaign took place in 2 separate periods, phase I in July and August and phase II in October and November. During the campaign, cosmic muons events were recorded in a slice corresponding to about $5 \%$ of the final CMS detector, with portions of all subdetectors included in phase I. In phase II, the tracker was replaced by instruments to map the magnetic field. The triggers provided by all 3 muon subsystems recorded over 230 million events, with both the electromagnetic calorimeter and the tracker in the readout [3].

Cosmic muons, which are freely available on the earth's surface, were used to test the CMS detector. The energy spectrum and intensity of cosmic muons at sea level cause them to appear in the CMS detector with high energy and high frequency. The distribution of the incident angle from vertical $\theta$ of $\operatorname{cosmic}$ muons is proportional to $\cos \theta \sin \theta$, which indicates that cosmic muons appear in the barrel region more often than in the endcap region. The energy spectrum is described by a power law $\sim E_{\mu}^{-2.7}$, and there are about $20 \%$ more $\mu^{+}$than $\mu^{-}$[2].

\subsection{Simulated samples}

The same simulated event sample as used in [5] was employed here. Four subsets of the simulated sample were selected to study the performance of cosmic muon reconstruction and manifest the difference with respect to the standard algorithm.

The first subset was selected to imitate the data taken during the MTCC phases I and II. Only the muon chambers and tracker modules used in the MTCC were activated in the reconstruction. A pseudo-trigger filter was applied to the simulated sample to mimic the trigger conditions used in run 2621 in order to take account of the effect of the trigger on the quality of events.

A second subset was selected from the first one by taking barrel-endcap overlapping tracks as a special case for demonstrating the differences between the different algorithms. The data subset included only those events with at least 1 DT segment and at least 1 CSC segment.

A third subset was also selected from the first one by choosing those events that contain both muon and tracker tracks. It was used to study the performance of the global cosmic muon reconstruction. Data taken during the MTCC phase I were used for the comparison.

A fourth subset was selected to study the performance of the reconstruction of traversing muons. There was no selection on chambers or pseudo-trigger filter applied. The subset included only those events that have 2 muon tracks reconstructed by the standalone muon reconstruction. The properties of the muon tracks and their 2 separate parts in different hemispheres were studied.

\section{Performance}

The performance of the cosmic muon reconstruction algorithm was studied under several scenarios with different data sets as described below. The following values are useful when considering the performance of muon reconstruction. The reconstructed transverse momentum resolution is obtained by a Gaussian fit to the distribution of

$\frac{q^{\mathrm{rec}} / p_{\mathrm{T}}^{\mathrm{rec}}-q^{\mathrm{sim}} / p_{\mathrm{T}}^{\mathrm{sim}}}{q^{\mathrm{sim}} / p_{\mathrm{T}}^{\mathrm{sim}}}$,

where $q^{\text {rec }}$ and $p_{\mathrm{T}}^{\text {rec }}$ are the charge and transverse momentum taken from the most accurate trajectory state of a reconstructed cosmic track, $q^{\text {gen }}$ and $p_{\mathrm{T}}^{\text {gen }}$ are the charge and transverse momentum of the simulated hit closest to the state. The reconstruction efficiency for each condition is defined separately for each case as listed in Table 1 . The purity is defined as the number of events with only 1 track reconstructed divided by the number of events with 1 or more tracks reconstructed, while there was only 1 muon track simulated for each event.

Since CMS software was under active development, the overall performance of the release used for this study had not achieved the full design performance as described in PTDR [7]. The results presented in this article can nevertheless be considered as a baseline of what CMS software can achieve. The performance of cosmic muon reconstruction is summarized in Table 1. Further detail is presented below. Figure 6 shows 2 examples of successfully reconstructed cosmic muon tracks. 
Table 1 Summary of the performance of cosmic muon reconstruction

\begin{tabular}{llcc}
\hline Event description & Efficiency definition & Efficiency & Purity \\
\hline MTCC (real data) & $\frac{N_{\text {tracks }}}{N_{\text {more than 1 segment }}}$ & $93 \%$ & - \\
MTCC (simulated data) & $\frac{N_{\text {tracks }}}{N_{\text {more than 1 segment }}}$ & $90 \%$ & $99 \%$ \\
MTCC overlap (real data) & $\frac{N_{\text {tracks }}}{N_{\text {more than } 1 \text { segment }}}$ & $90 \%$ & - \\
MTCC global (real data) & $\frac{N_{\text {global muons }}}{N_{\text {tracks in both muon and tracker systems }}}$ & $>46 \%$ & - \\
Traversing tracks (simulated data) & $\frac{N_{\text {tracks }}}{N_{2 \text { tracks in opposite hemispheres }}}$ & $85 \%$ & $99 \%$ \\
\cline { 2 - 3 } Beam-halo (simulated data) & $\frac{N_{\text {tracks }}}{N_{\text {more than } 1 \text { segment }}}$ & $99 \%$ & $93 \%$ \\
\hline
\end{tabular}

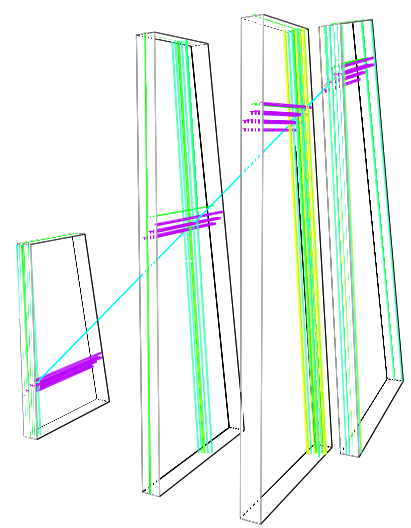

(a)

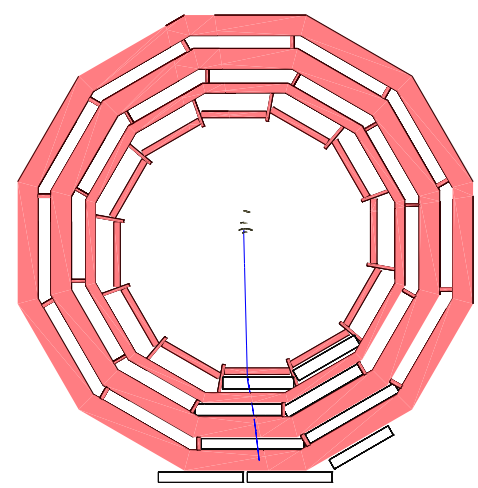

(b)

Fig. 6 IGUANA [11] screen-shots of (a) a reconstructed track passing through CSC layers (Run 2621, Event 22); and (b) the first reconstructed track passing through the tracker, DTs, and CSCs (Run 2621, Event 68563)

\subsection{Algorithm performance using MTCC simulated and real data}

\subsubsection{Reconstruction and analysis chain}

The electronic signals processed by the CMS front-end electronics and data acquisition (DAQ) system were translated into C++ classes, called "digis", by software packages called "unpackers." Then the local reconstruction packages built hits and segments using the digis in each muon chamber. The RPC subsystem was included in the reconstruction of the simulated sample but not included in the MTCC real data because the RPC subsystem was not included in the global DAQ for MTCC phase I and for most runs in MTCC II. However, this difference should not significantly affect the results.

After local reconstruction in each subsystem, the cosmic muon reconstruction starts with hits and segments to build trajectories as described in Sect. 3.

\subsubsection{Results}

Figure 7(a) and (b) show the $p_{\mathrm{T}}$ distributions of reconstructed cosmic tracks from simulated data and real data, which are in agreement. The reconstruction efficiency is defined as the number of events with the track reconstructed successfully divided by the number of events with 2 or more track segments with measurements in all space coordinates built in different DT or CSC chambers. The measured efficiency for the simulated sample was $90 \%$, while for MTCC data it was $93 \%$. The measured efficiencies with MTCC data with DTs only, with CSCs only, and with DT-CSC overlap were $97 \%, 81 \%$, and $90 \%$, respectively. These results are consistent with another study using DTs only [5]. The measured purity with simulated data was $99 \%$.

The $p_{\mathrm{T}}$ resolution obtained with the standard muon reconstruction was about $34 \%$, while with the cosmic muon reconstruction it was about $26 \%$. With the cosmic muon reconstruction, the resolution was about $38 \%$ using CSC only and about $24 \%$ using DT only. These results are also consistent with [5]. The overall resolution is better with the DT system only than with the CSC system only because trajectories traveling through the DT system usually form larger angles from the magnetic field, rendering a better measurement of the curvature. 


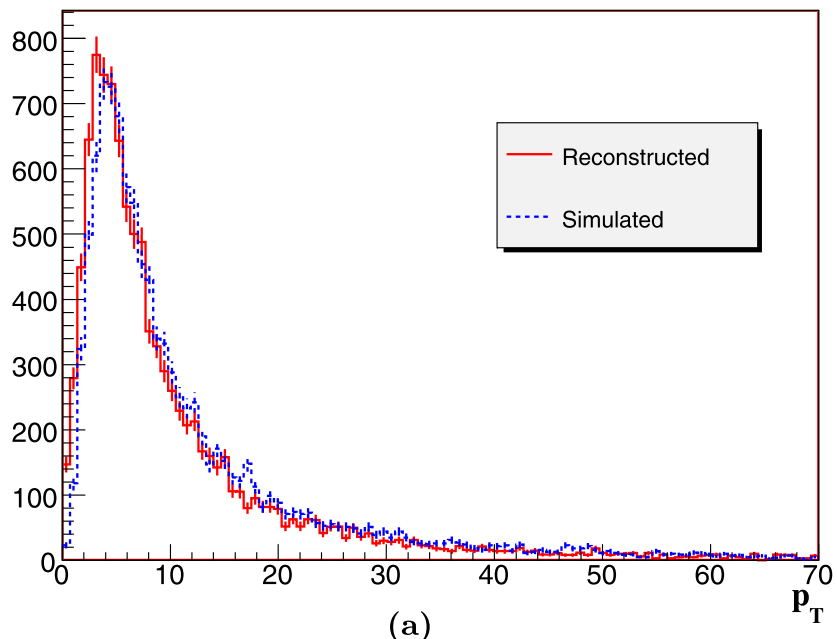

(a)

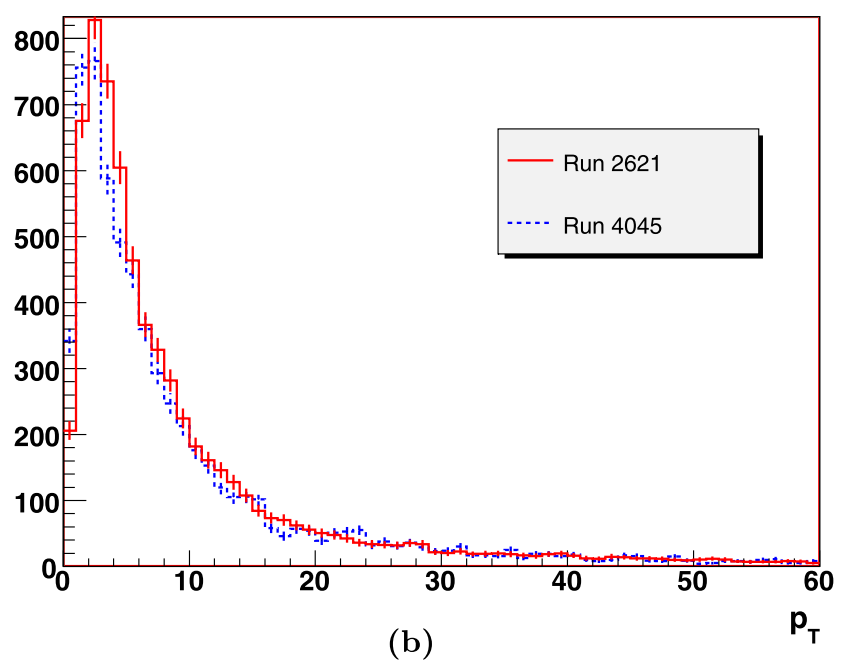

Fig. 7 (a) The $p_{\mathrm{T}}$ distribution at the first measurement of the simulated and reconstructed cosmic muon tracks. (b) The $p_{\mathrm{T}}$ distribution at the first measurement of cosmic muon tracks of real data taken from MTCC runs 2621 and 4045

\subsubsection{Performance using barrel-endcap overlap tracks}

The special case of barrel-endcap overlap tracks (see Fig. 1(f)) is an excellent example to show the difference between the cosmic and the standard reconstruction approach in each step: seeding, navigation, and trajectory building. To demonstrate the effect of the difference of each component, the same data are processed with 4 sets of algorithms: (1) standard standalone reconstruction, (2) standard method with cosmic muon seed generation algorithm, (3) standard method with cosmic muon seed generation algorithm and direct muon navigation method, and (4) full cosmic muon reconstruction.

Figure 8 illustrates that the number of hits contained in each track and the total number of reconstructed tracks increase from methods (1) to (4) as more cosmic muon optimized components are involved.

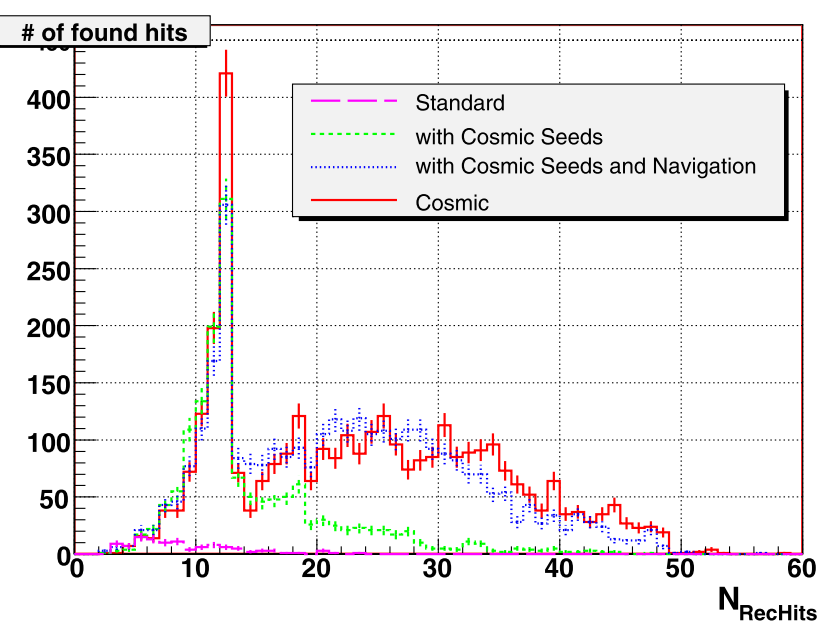

Fig. 8 Number of hits contained in each track by (1) standard standalone reconstruction, (2) standard method with cosmic muon seed generation algorithm, (3) standard method with cosmic muon seed generation and direct muon navigation algorithm, and (4) cosmic muon reconstruction

\subsection{Performance of global cosmic muon reconstruction in MTCC}

The first reconstructed muon trajectory passing through the Tracker, DTs, and CSCs (Fig. 6(b)) was reported shortly after the MTCC phase I.

The efficiency of global cosmic muon reconstruction is defined as the number of events with the global cosmic muon track built successfully over the number of events with 1 standalone cosmic muon track and 1 tracker track. From the data of run 2377, the measured efficiency by this definition was about $46 \%$. The inefficiency is mainly due to tracker and muon system tracks that do not match each other. There are several reasons for this inefficiency. First, the tracks in the tracker and muon systems could be induced by different cosmic muons arriving in the same time window. Second, there is not enough information available about the alignment between the tracker system and the rest of the CMS detector. Third, the ideal 4-T magnetic field setup was adopted during the reconstruction, while the magnetic field was 3.8 $\mathrm{T}$ when collecting the data. The effect of this difference was not obvious within each subdetector but became more significant when comparing tracks inside and outside the superconducting solenoid. Overall, the real efficiency should be much higher than this reported value. Figure 9 also shows the $p_{\mathrm{T}}$ distribution of global cosmic muons and their corresponding tracks in the muon and tracker systems. The measurement of track $p_{\mathrm{T}}$ in the tracker system is inaccurate because many tracks contain hits in only 2 layers.

\subsection{Performance using traversing muons}

It is possible to observe muons that traverse the whole CMS detector, as illustrated in Fig. 10. With the algorithm de- 


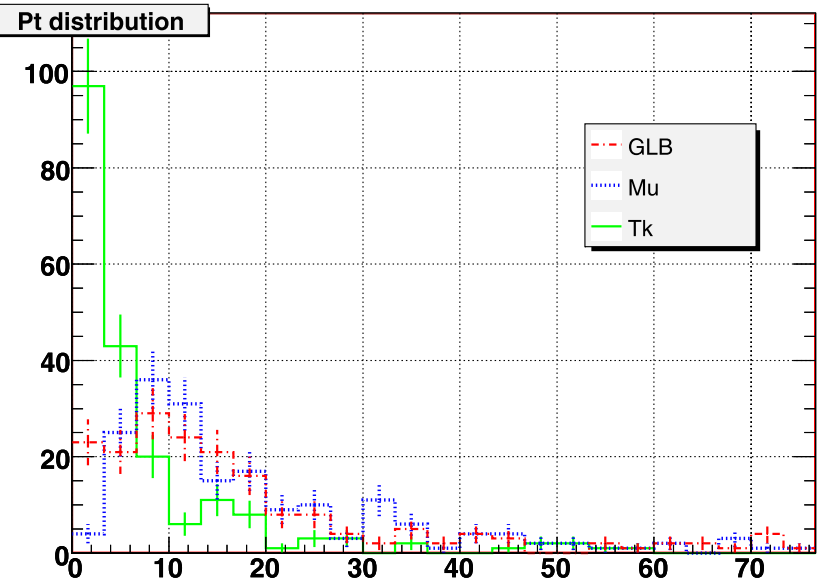

Fig. 9 The $p_{\mathrm{T}}$ distribution of tracks in the muon system, tracker system, and combined system, with data from run 2621

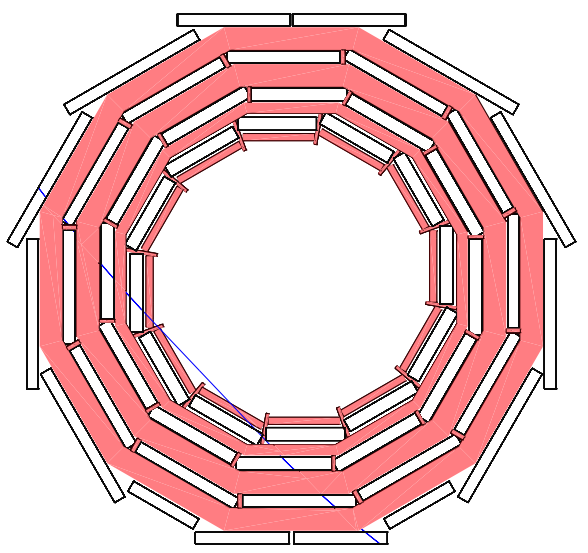

Fig. 10 IGUANA screen-shot of a reconstructed muon track that passes through 2 hemispheres of the detector

scribed here, all hits from both hemispheres of the detector can be used in a single trajectory, which in turn allows for a more precise momentum measurement and provides an excellent tool for alignment.

The efficiency to reconstruct traversing muons is defined as the number of events containing a track passing through 2 hemispheres divided by the number of events containing 2 separate standalone tracks in the 2 hemispheres. The measured efficiency is about $85 \%$. Because more hits over a larger space are included in traversing trajectories, the $p_{\mathrm{T}}$ resolution of traversing tracks is better than the average value in the MTCC. Figure 11 shows that traversing tracks contain more hits than non-traversing ones. Figure 12 shows that the $p_{\mathrm{T}}$ resolution is $9 \%$ for those trajectories with more than 46 hits, while for those with fewer than 46 hits it is $25 \%$.

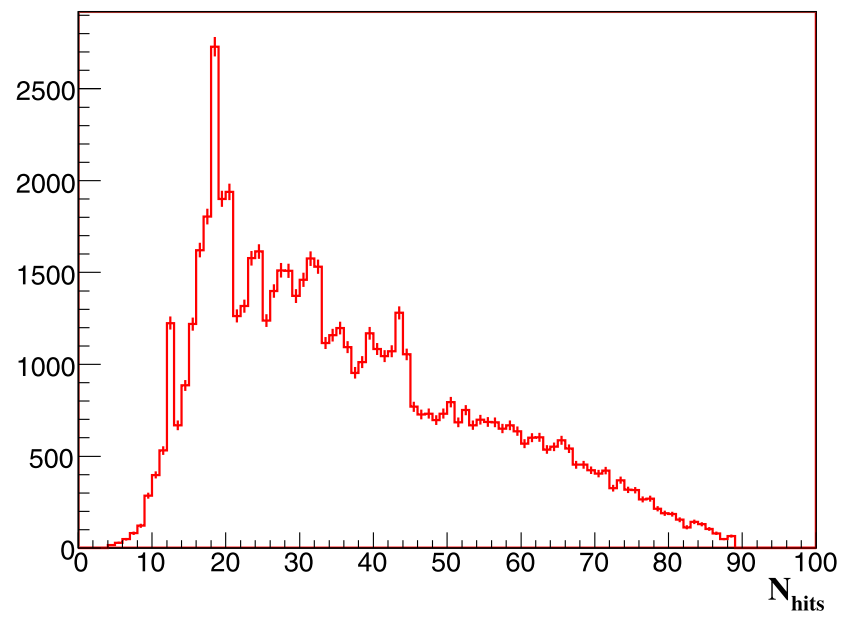

Fig. 11 Number of hits contained in each track by cosmic muon reconstruction. The long tail of tracks with more than 46 hits represents successfully reconstructed traversing muons

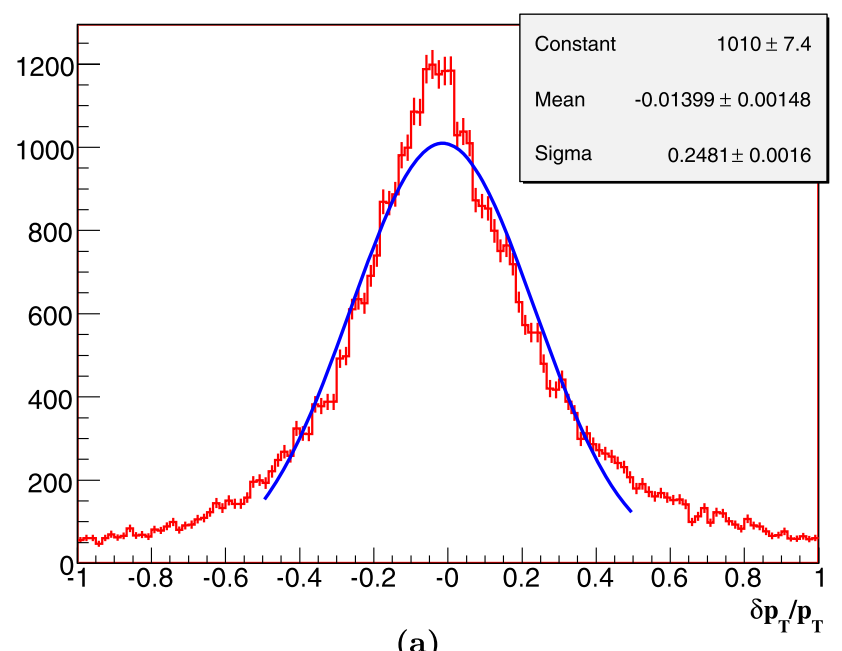

(a)

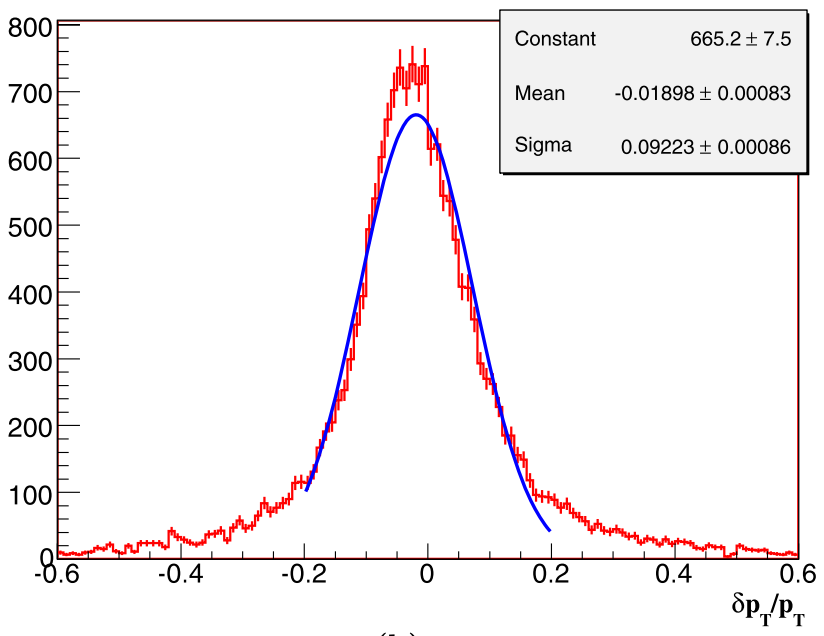

(b)

Fig. 12 (a) The $p_{\mathrm{T}}$ resolution of non-traversing muons with fewer than 46 hits in the first trajectory states is about $25 \%$. (b) The $p_{\mathrm{T}}$ resolution of traversing muons with more than 46 hits in the first trajectory states is about $9 \%$ 


\section{Strategy and performance of beam-halo muon reconstruction}

Beam-halo muons are machine-induced particles that travel along the beam line from outside of the detector. In CMS software, beam-halo muons can be reconstructed by the same software package and configuration used for cosmic muons. When the $|\eta|$ value of the momentum of a trajectory seed in the endcap region exceeds a given threshold (currently set as 4.5), it is identified as a beam-halo muon. In this case, all barrel layers are skipped when asking for compatible layers in the navigation step, because beam-halo muons will pass through the entire sensitive zone of the barrel DT and RPC chambers, which creates a large amount of charge to be deposited and decreases the chamber efficiency [7]. Although not all beam-halo muons arrive at the second endcap as shown in Fig. 13, the layers in the second endcap are still chosen as compatible layers. The compatible layers are ordered as outside-in on one end and inside-out on the other. The navigation direction is flipped from outside-in to inside-out when the endcap region changes during building trajectory.

Since the $p_{\mathrm{T}}$ of beam-halo muons is too low to be used for performance studies, the momentum resolution was studied instead. With a privately-generated sample containing 3000 events of beam-halo muons from the positive $z$ direction only, the measured momentum resolution for reconstructed beam-halo muons is about $41 \%$, as illustrated in Fig. 14(a). As demonstrated in Fig. 14(b), a large discrepancy exists in the low momentum region as expected since the directions of beam-halo muons are almost parallel to the magnetic field and most of the low momentum beam-halo

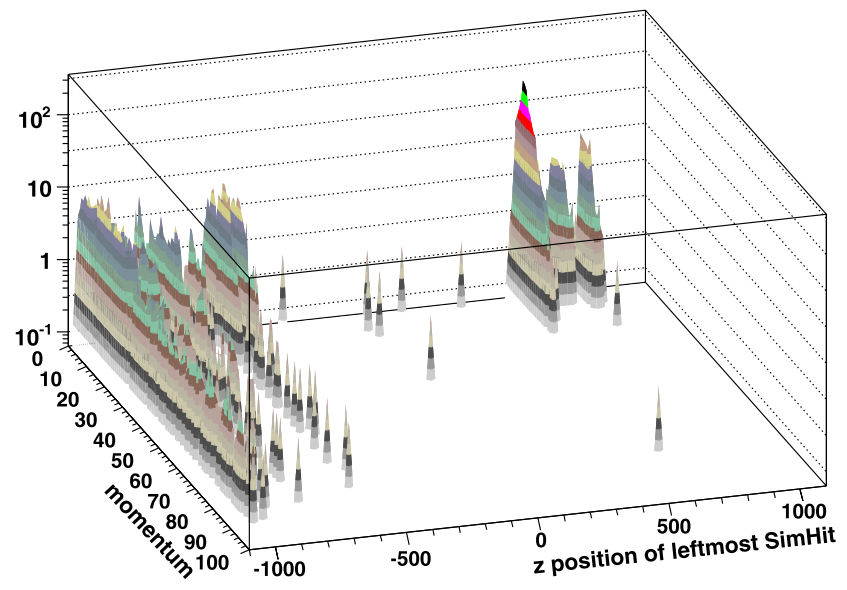

Fig. 13 Distribution of the $z$-component of the leftmost simulated hit and momentum of the injected beam-halo muon. The beam-halo muons were generated to come from the right hand side (from positive $z$ and corresponding to the LHC beam 1). The peaks on the right side of the plot represent those beam-halo muons that failed to arrive at the second endcap. Almost all muons with $p>25 \mathrm{GeV}$ reached the second half

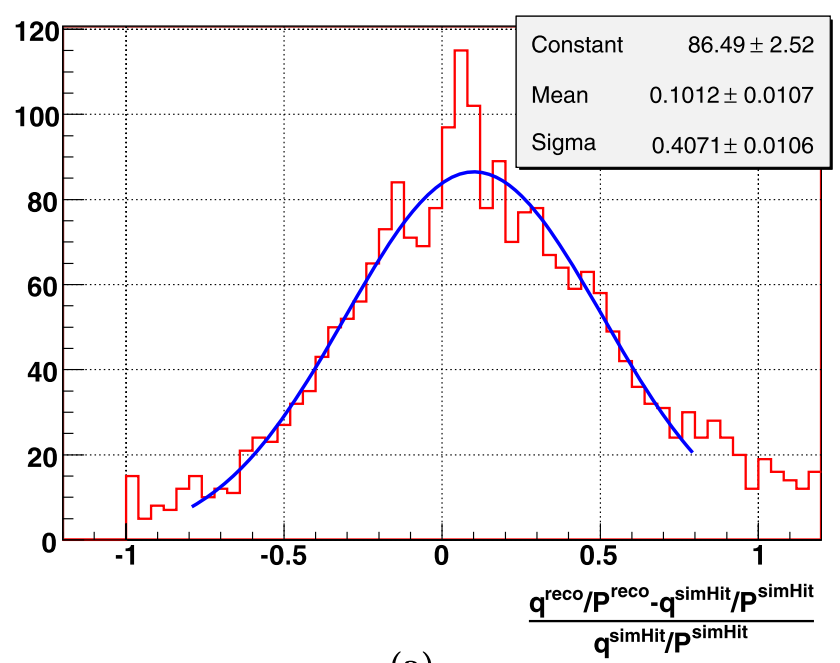

(a)

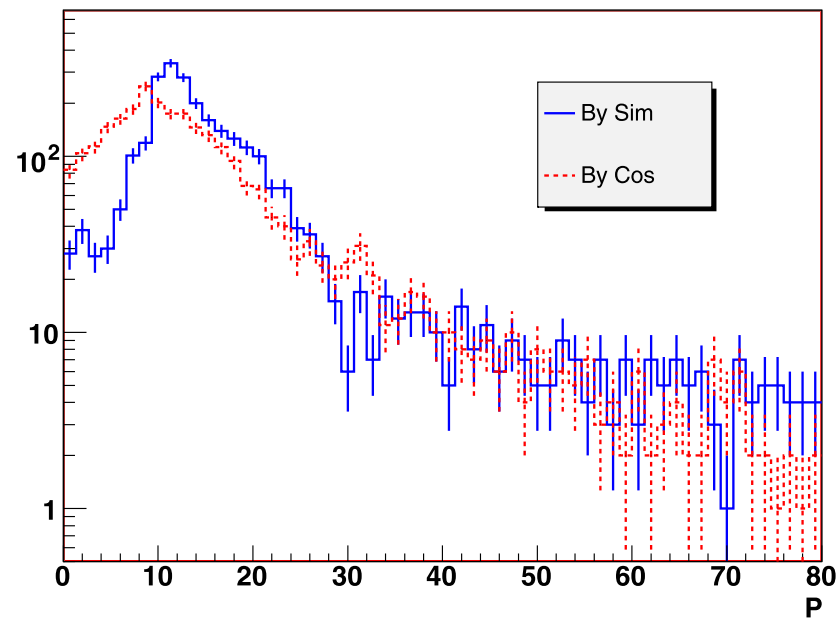

(b)

Fig. 14 (a) The momentum resolution of beam-halo muons at the first measurement is about $41 \%$. (b) Momentum distribution at the first measurement of the simulated and reconstructed beam-halo muon tracks

muons can only penetrate a few layers. The reconstruction efficiency, defined as the number of events with track reconstructed successfully divided by number of events with 2 or more track segments built in CSC system, is about $99 \%$.

\section{Summary}

In this article we have described a new algorithm designed to reconstruct cosmic muons and discussed the different reconstruction strategy compared to the standard muon reconstruction algorithm. The new cosmic muon reconstruction algorithm works efficiently for both cosmic muons and beam-halo muons. A full detector simulation and reconstruction analysis was carried out to validate the performance. In addition, data taken during the MTCC were compared to simulated cosmic data, and good agreement be- 
tween simulated and reconstructed results was observed. The presented cosmic muon reconstruction software provides a powerful tool to utilize cosmic and beam-halo muons for synchronization and alignment during the commissioning of the CMS detector and the initial data taking period at the LHC.

Acknowledgements We would like to thank the muon physics object group for their collaboration in the software development and integration; all the developers who contributed to the CMS software project; Emanuela Barberis, Ugo Gasparini and Martijn Mulders for their help in the analysis of MTCC data; all the people whose work made the MTCC a big success; Darin Acosta, Dan Holmes, and Marcello Maggi for discussions on beam-halo triggers and detector readout; Richard Breedon for valuable suggestions on the organization and writing when reviewing the note; and Adam Everett for checking the manuscript.

\section{References}

1. CMS Collaboration, Technical proposal, CERN/LHCC 94-38

2. Paticle Data Group, W.M. Yao et al., J. Phys. G 33, 1 (2006)

3. CMS Collaboration, CMS NOTE 2007/005

4. V. Drollinger, CMS NOTE 2005/012

5. P. Biallass et al., CMS NOTE 2007/024

6. N. Amapane et al., CMS NOTE 2007/034

7. CMS Collaboration, CMS Physics TDR, vol. 1, CERN/LHCC 2006-001

8. E. Barberis et al., CMS NOTE 2006/012

9. R. Frühwirth, Nucl. Instrum. Methods A 262, 444 (1987)

10. D. Benedetti et al., CMS NOTE 2007/030

11. IGUANA Webpage. http://iguana.web.cern.ch/iguana 